Asian Journal of Information Technology 10 (7): 271-276, 2011

ISSN: $1682-3915$

(C) Medwell Journals, 2011

\title{
Smart Software Systems: Its Evolution and Application
}

\author{
Akazue Maureen \\ Department of Mathematics and Computer Science, Delta State University, Abraka, Nigeria
}

\begin{abstract}
The primary vehicle in the quest for intelligent software has been the gradual recognition of the central role played by data and information rather than the logic and functionality of the application. Smart software agents have the capability of reacting to its environment, being autonomous, goal-orientation and persistence as well as exhibit flexible behavior, reactive and proactive nature with some degree of social ability. This study focused on the evolution of smart software agents; four essential types of intelligent software agents; the importance of smart software agents. Furthermore, these capabilities were examined by designing an ecommerce website for Delta State University bookshop which was test-run by different users. It was concluded that smart software agents have come to stay as they are predefined clients within user systems and perform internal functions that are necessary for the particular user to deliver its services.
\end{abstract}

Key words: Software agents, artificial intelligence, intelligent agents, expert systems, smart software agents, Nigeria

\section{INTRODUCTION}

The concept of an agent can be traced back to Hewitt's Actor model (Hewitt, 1997). A self-contained, interactive and concurrently-executing object, possessing internal state and communication capability. Software agent systems are a direct evolution from Multi-Agent Systems (MAS) which evolved from Distributed Artificial Intelligence (DAI), Distributed Problem Solving (DPS) and Parallel AI (PAI) thus, inheriting all characteristics (good and bad) from DAI and $\mathrm{AL}$ (Phillips-Wren and Jain, 2005). The primary vehicle in the quest for intelligent software has been the gradual recognition of the central role played by data and information, rather than the logic and functionality of the application.

Thus, the three milestones in this evolution have been: the separation of data management from the internal domain of the application; the development of standard data exchange protocols such as the Extensible Markup Language (XML) and the ability to build information models that are rich in relationships and are thereby capable of supporting the automated reasoning capabilities of software agents (Phillips-Wren and Jain, 2005).

Nwana (1996), Wooldridge (2002, 2003), Russell and Norvig (2003) and Phillips-Wren and Jain (2005) have proposed different definitions of agents and these commonly include concepts such as persistence (code is not executed on demand but runs continuously and decides for itself when it should perform some activity), autonomy (agents have capabilities of task selection, prioritization, goal-directed behavior, decision making without human intervention), social ability (agents are able to engage other components through some sort of communication and coordination, they may collaborate on a task), reactivity (agents perceive the context in which they operate and react to it appropriately).

This study focuses on the evolution of smart software agents; four essential types of intelligent software agents; the importance of Smart Software agents and their capabilities were examined by designing an ecommerce website for Delta State University bookshop which was test-run by different users.

\section{LITERATURE REVIEW}

Software agent can be termed to be a program that acts for a user or other program in a relationship. In other words it has the authority to decide which (and if) action is appropriate. The idea is that most agents are not strictly invoked for a task but activate themselves (Artificial intelligence). 


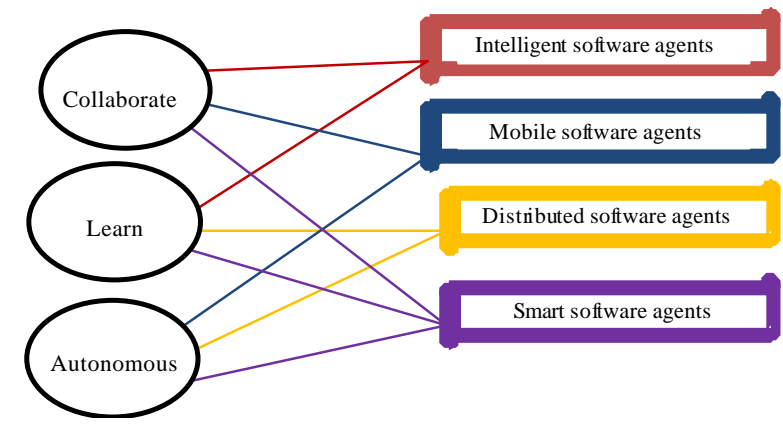

Fig. 1: Definition of software agents

These software agents are called intelligent agents when they exhibit some aspect of learning and reasoning, autonomous agents when they modify the way of achieving their objective, distributed agents when being executed on physically distinct computer, multi-agent systems when the distributed agents communicate with other agents in other to achieve an objective, mobile agents when they have the capability to relocate their execution onto different processors and Smart software agents when they have the capability to reason, learn, work together and modify ways of achieving objectives. Therefore, smart software agents (Fig. 1) have the capability of reacting to its environment being autonomous, goal-orientation and persistence as well as exhibit flexible behavior, reactive and proactive nature with some degree of social ability.

Distinguishing intelligent (smart) software agents from intelligent agents: Generally, smart agents are different from arbitrary programs Franklin and Graesser (1996), objects (Wooldridge, 2002), expert systems ((Wooldridge, 2003) and intelligent agents (http://en.wikipedia.org/ wiki/File:IntelligentAgent-SimpleReflex.png; Russell and Norvig, 2003).

\section{MATERIALS AND METHODS}

The fact-finding techniques used in information gathering were document review, research and site visit as well as designing an ecommerce website for Delta State University bookshop which was test-run by different users to demonstrate the role of software agents in delivering services.

Intelligent software agents (smart software agents): The internet allows different activities to be performed and thus different smart software agents are needed to achieve such purposes. According to Haag (2006), there are only four essential types of intelligent software agents; buyer agents or shopping bots, user or personal agents, monitoring-and-surveillance agents and data mining agents.

The buyer agents (shopping bots): Retrieve information about goods and services by using the technology known as collaborative filtering (amazon.com).

User agents (personal agents): They are intelligent agents that helps perform the tasks of checking e-mail and sending alert when important emails arrives, play computer games as the opponent, assemble customized news reports, find information on the choice of subject, fill out forms on the web automatically and stores the information for future reference, discuss topics with the user ranging from deepest fears to sports, facilitate with online job search duties by scanning known job boards and sending the resume to opportunities who meet the desired criteria and profile synchronization across heterogeneous social networks.

Monitoring and surveillance (predictive) agents: These are used to observe and gives report on equipment like the computer systems. These agents keep track of company inventory levels, observe competitors' prices and relay them back to the company, watch stock manipulation by insider trading and rumors service monitoring as well as monitor complex computer networks that can keep track of the configuration of each computer connected to the network.

Data mining agents: Uses information technology to find trends and patterns in an abundance of information from many different sources. This agent operates in a data warehouse discovering information and can also detect major shifts in trends or a key indicator and can detect the presence of new information and alert one.

The importance of smart software agents: Advancements in computer technology have made it possible to store vast amounts of data in electronic form. Typically, data file and database management methodologies focused on the storage, retrieval and manipulation of data transactions rather than the context within which the collected data would later become useful in planning, monitoring, assessment and decision-making tasks (Pohl, 2004). It relates to the complexity of networked 
computer and communication systems and the increased reliance of organizations on the reliability of such information technology environments as the key enabler of their effectiveness, profitability and continued existence. For instance is the TEGRID Semantic Web Application by Gollery and Pohl (2002) shown in Fig. 2 and 3 .

Application of smart software agents: The software agents are innovative technologies that may offer various

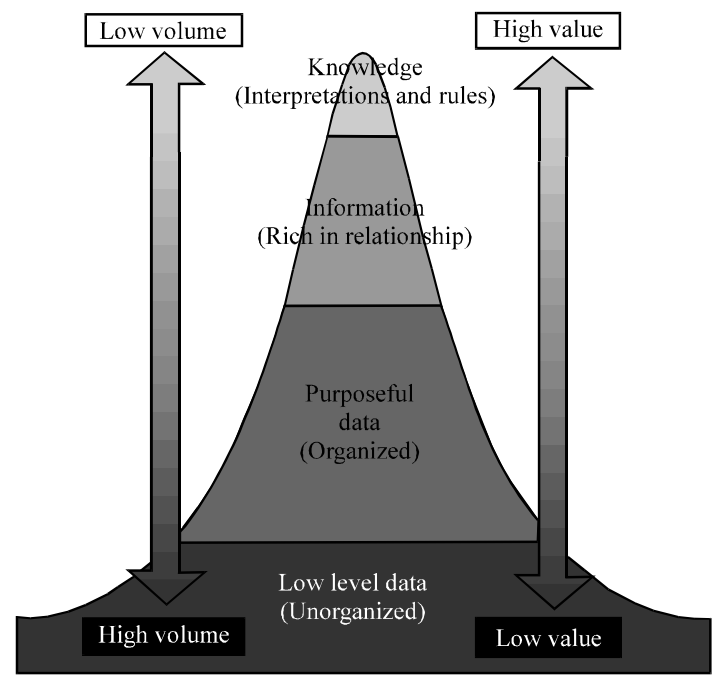

Fig. 2: Transition from data to knowledge benefits to their end users by automating complex or repetitive tasks (Serenko and Detlor, 2004.) Software agents are able to quickly search the internet, identify the best offers available online and present this information to the end users.

Therefore, users may not need to manually browse various websites of individual merchants; they are able to locate the best deal in a matter of seconds.

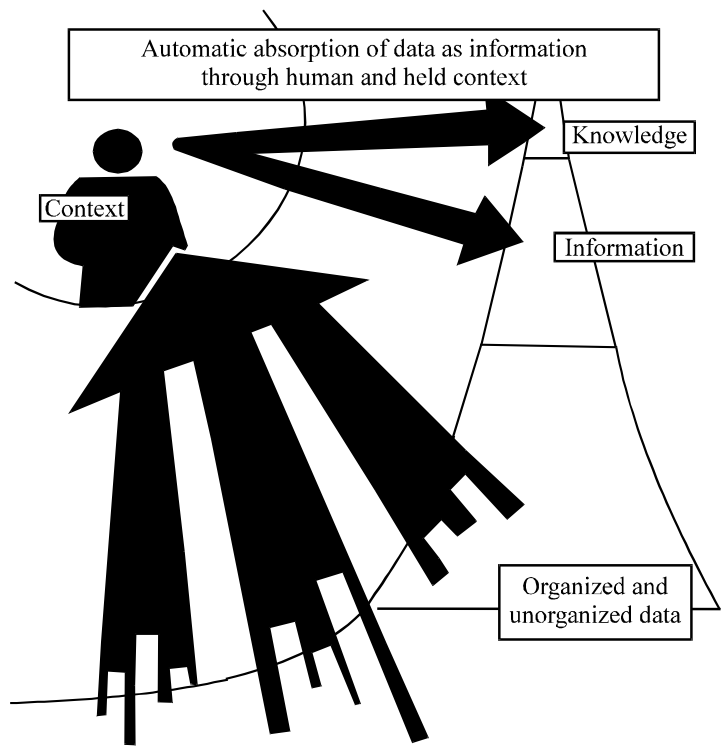

Fig. 3: Human interpretation of data

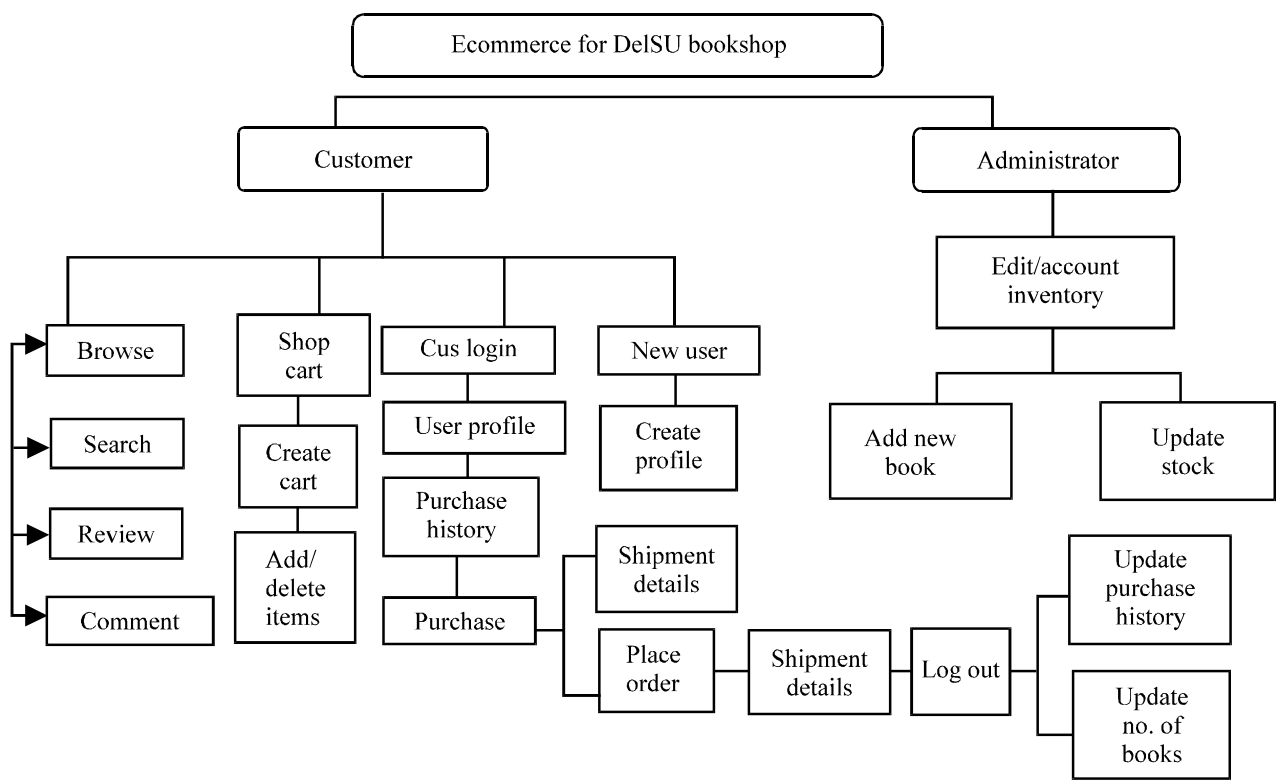

Fig. 4: E-commerce for DelSU bookshop 


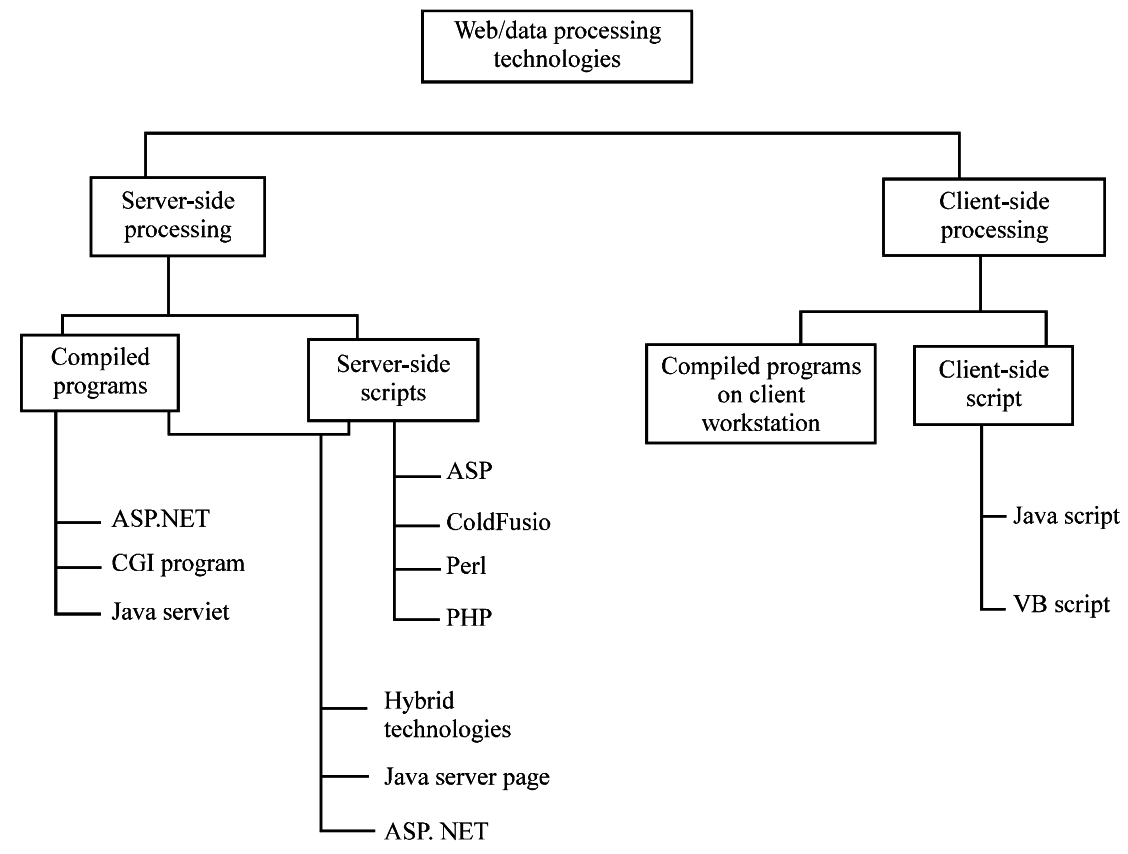

Fig. 5: Web page programming option

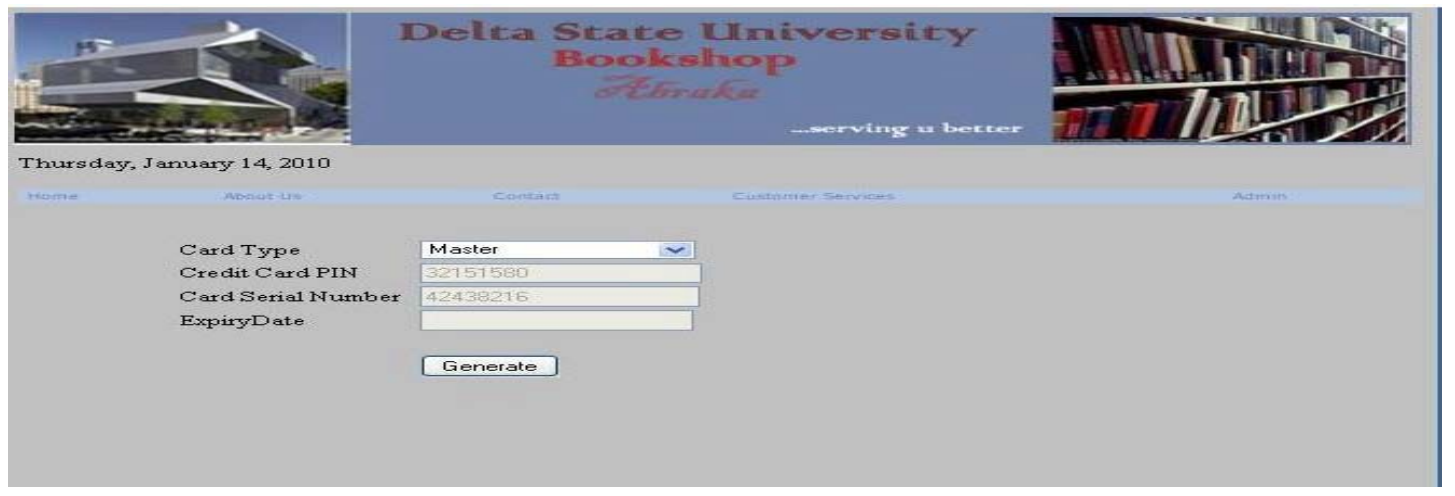

Fig. 6: Generation of Master Credit card

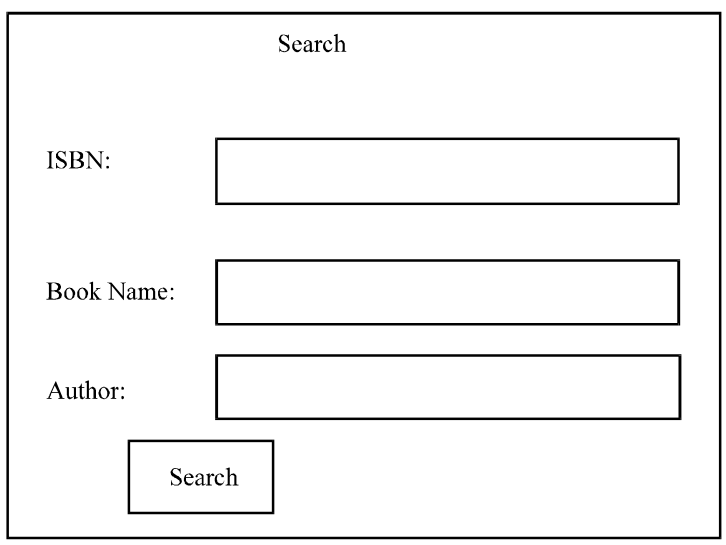

Fig. 7: For searching the books in the store

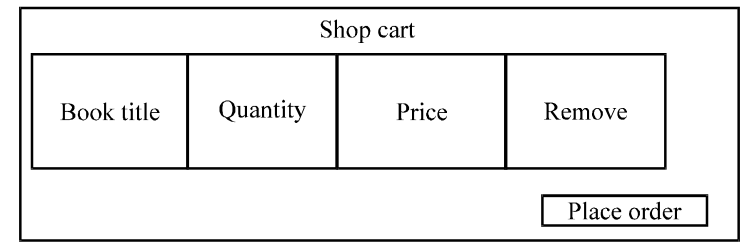

Fig. 8: Shop cart for the user

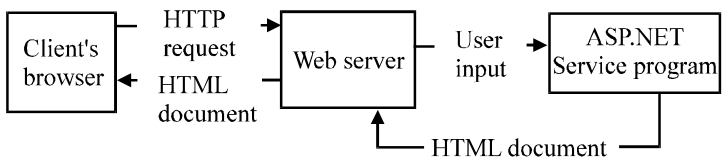

Fig. 9: Compiled server programs flow chart 
Asian J. Inform. Technol., 10 (7): 271-276, 2011

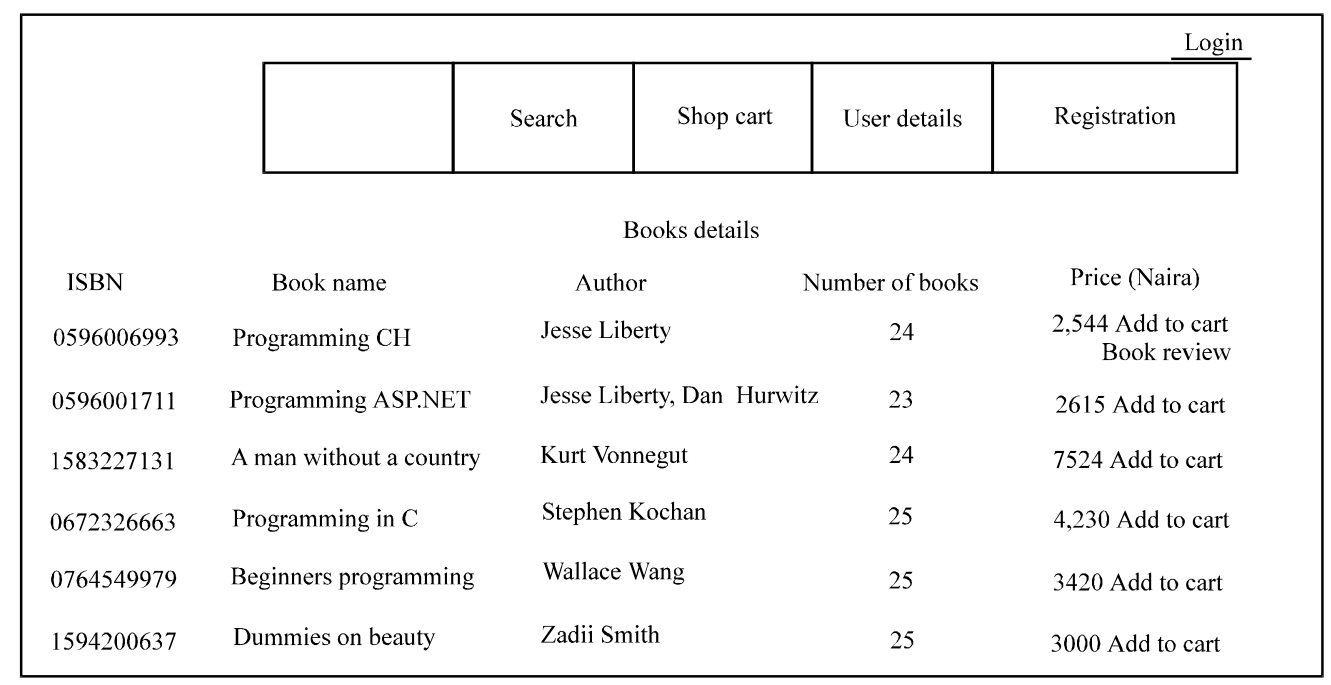

Fig. 10: Book details

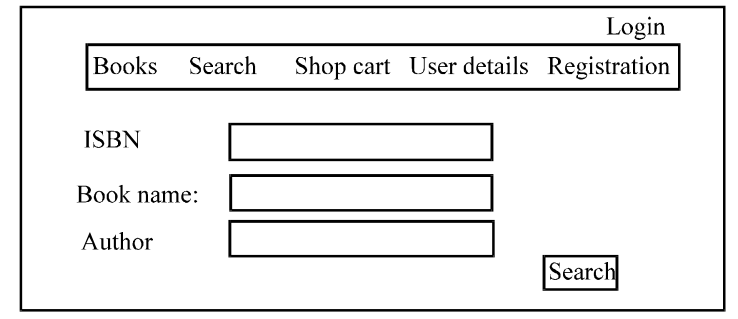

Fig. 11: Search for books

In illustrating the role of smart software agents an ecommerce website for Delta State University bookshop was designed and test-run (Fig. 4-11). The program creates an HTML document to be sent to the client browser.

\section{RESULTS AND DISCUSSION}

According to Gollery and Pohl (2002) and Pohl (2004), a more complete information management environment considers data to be the bottom layer of a three-layer architecture, namely; data layer (that integrates heterogeneous data sources into accessible and purposefully ordered data), mediation layer (that defines the logical data models, data transfer formats, data transformation rules and also serves as a translation facility for bridging between data with structural relationships) and Information layer (that consists of many functionally oriented planning and decision assistance software applications).

In such a three-layered information management environment, the mediation layer continuously populates the information models of the applications in the information layer with the data changes that are fed to it by the data layer. This in turn automatically triggers the reasoning capabilities of the software agents. Thus, the agents can be looked upon as intelligent dynamic tools that continuously monitor changes in the real world. They utilize their reasoning and computational capabilities to generate and evaluate courses of action in response to both real world events and user interactions as a result the human user is relieved of many of the lower level filtering, analysis and reasoning tasks that are a necessary part of any useful planning and problem solving process.

In the test-run of the DelSU bookshop site, the capabilities of the smart software agent were observed through the ability of an executing software entity to extend its information model by gaining access to portions of the information model of another executing software entity; ability of several semantic web services to collaboratively assist each other and human users during time of critical decision-making processes; ability of a software agent to automatically reason about events in near real-time under time critical conditions and ability of a semantic web service to create an agent to perform specific situation monitoring and reporting functions.

The reasoning capabilities are performed by software agents that are components of the players (Wooldridge and Jennings (1995). In other words, agents are predefined clients within computer systems and perform internal functions that are necessary for the particular system to deliver its services.

Disadvantages/Pitfalls of smart software agents: The implementation of agents also requires additional 
resources from the companies, places an extra burden on their networks and requires new security procedures. The cultural effects of the implementation of software agents include trust affliction, skills erosion, privacy attrition and social detachment. Some users may not feel entirely comfortable fully delegating important tasks to software applications.

In order to act on a user's behalf, a software agent needs to have a complete understanding of a user's profile including his/her personal preferences. This in turn may lead to unpredictable privacy issues. When users start relying on their software agents more, especially for communication activities, they may lose contact with other human users and look at the word with the eyes of their agents. It is these consequences that agent researchers and users need to consider (Serenko et al., 2007).

\section{CONCLUSION}

Computers have been shown to have learning-like capabilities and computers can discover information through associations and pattern matching (Phillips-Wren and Jain, 2005). Furthermore, there is an urgent need for intelligent computer capabilities due to the mounting expectations of accuracy, quality and timeliness in a globally connected environment of rapidly increasing complexity.

It is clear therefore that the continued expansion of networks such as the Internet and its successors will provide seamless connectivity among countless nodes on a global scale and smart software agents will always be a necessity in the networked environment.

\section{REFERENCES}

Franklin, S. and A. Graesser, 1996. Is it an agent, or just a program: A taxonomy for autonomous agents. Proceedings of the 3rd International Workshop on Agent Theories, Architectures and Languages, August 12-13, 1996, Springer-Verlag, Berlin, pp: 1-10.
Gollery, S. and J. Pohl, 2002. The TEGRID semantic web application. A Demonstration System with Discovery, Reasoning and Learning Capabilities, Office of Naval Research (ONR), Workshop Series on Collaborative.

Haag, S., 2006. Management Information Systems for the Information Age. McGraw Hill Co., New York, pp: 224-228.

Hewitt, C., 1997. Viewing control structures as patterns of passing messages. Artif. Intell., 8: 323-364.

Nwana, H.S., 1996. Software agents: An overview. Knowledge Eng. Rev., 11: 1-40.

Phillips-Wren, G.E. and L.C. Jain, 2005. Intelligent Decision Support Systems in Agent-Mediated Environments. IOS Press, Amsterdam, ISBN-13: 9781586034764, pp: 3-34.

Pohl, J., 2004. The evolution of intelligent computer software and the semantic web. Proceedings of the 16 th International Conference on Systems Research, Informatics and Cybernetics, July 29-August 5, 2004, Baden-Baden, Germany, pp: 11-34.

Russell, S. and P. Norvig, 2003. Artificial Intelligence: A Modern Approach. 2nd Edn., Prentice Hall, New York, ISBN-13: 978-0137903955, Pages: 1132.

Serenko, A. and B. Detlor, 2004. Intelligent agents as innovations. Artif. Intell. Soc., 18: 364-381.

Serenko, A., U. Ruhi and M. Cocosila, 2007. Unplanned effects of intelligent agents on internet use: Social Informatics approach. Artif. Intell. Soc., 21: 141-166.

Wooldridge, J.M., 2002. Econometric Analysis of Cross Section and Panel Data. MIT Press, Cambridge, MA., ISBN-13: 9780262232197 , Pages: 752.

Wooldridge, J.M., 2003. Cluster-sample methods in applied econometrics. Am. Econ. Rev., 93: 133-138.

Wooldridge, M. and N.R. Jennings, 1995. Intelligent agents: Theory and practice. Knowledge Eng. Rev., 10: $115-152$. 